\section{PARTIAL CONGENITAL BOWEL OBSTRUCTION BY DUODENAL ATRESIA WINDSOCKS TYPE: CASE REPORT}

\section{Molinaro, F. Mariscoli, M. Sica, E. Bindi, R. Angotti, M. Messina}

Division of Pediatric Surgery, Department of Medical Science, Surgery and Neuroscience, University of Siena, Italy

\section{Introduction}

Duodenal atresia Windsocks type is a rare condition of congenital bowel obstruction. Thanks to recent technological advancements of prenatal diagnosis it is possible to make a diagnosis of duodenal atresia with high degree of certainty through the radiological sign of "double bubble", but up to date it is not yet possible to identify the type of duodenal atresia. We report the case of a patient with prenatal diagnosis of "double bubble". The patient had no other concomitant malformations.

\section{Case Report}

The patient came to our attention after prenatal ultrasound that showed a picture of double bubble. At the 27 th week of gestation we performed fetal MRI that confirmed the US pattern of double bubble but it did not identify with certainty the type of duodenal atresia. At birth the patient underwent GI rx examination that showed a picture of partial duodenal obstruction compatible with the Windsocks type. On the following day, we performed endoscopy which showed the presence of duodenal membrane, so the patient underwent surgical treatment with a longitudinal duodenal incision in order to treat the wind-sock membrane. After one month a further Upper-GI rx examination showed a regular transit of the contrast. Four months after the first operation the patient underwent new surgical treatment for bowel obstruction by adhesions. The operation was successful and the patient had a complete recover.

\section{Conclusions}

Patients with prenatal diagnosis of "double bubble" require a multidisciplinary approach for proper clinical management. Unfortunately it is not currently possible to identify with certainty by prenatal ultrasound the type of duodenal atresia, but in case of incomplete bowel occlusion, the possibility of an atresia Windsocks type should always be considered, especially for setting the right surgical approach. 